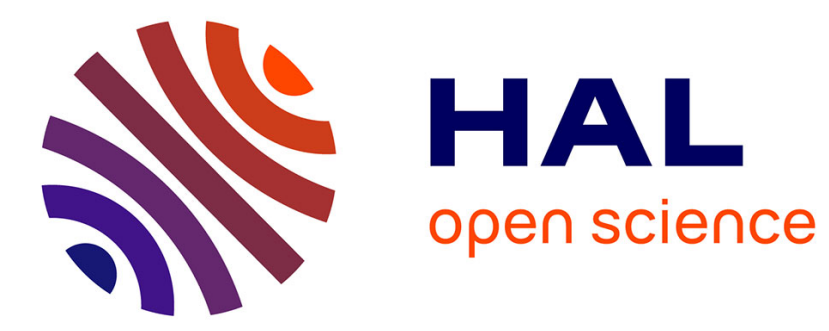

\title{
Vivre le vieillir : autour du concept de déprise
}

Anastasia Meidani, Stefano Cavalli

\section{To cite this version:}

Anastasia Meidani, Stefano Cavalli. Vivre le vieillir : autour du concept de déprise. Gérontologie et Société, 2018, Vivre le vieillir : autour du concept de déprise, 40 (155), pp.9-23. hal-02048333

\section{HAL Id: hal-02048333 \\ https://hal.science/hal-02048333}

Submitted on 25 Feb 2019

HAL is a multi-disciplinary open access archive for the deposit and dissemination of scientific research documents, whether they are published or not. The documents may come from teaching and research institutions in France or abroad, or from public or private research centers.
L'archive ouverte pluridisciplinaire HAL, est destinée au dépôt et à la diffusion de documents scientifiques de niveau recherche, publiés ou non, émanant des établissements d'enseignement et de recherche français ou étrangers, des laboratoires publics ou privés. 


\section{Vivre le vieillir : autour du concept de déprise}

Anastasia MEIDANI

Maîtresse de Conférences en sociologie, Laboratoire Interdisciplinaire Solidarités, Sociétés, Territoires (LISST), Université Toulouse - Jean Jaurès

Stefano CAVALLI

Professeur de sociologie, Scuola universitaria professionale della Svizzera italiana (SUPSI). Anastasia MEIDANI \& Stefano CAVALLI

Né à Toulouse, il y a déjà plus de trente ans, le concept de déprise se veut un outil analytique visant à rendre compte de l'expérience du vieillir. Serge Clément, Marcel Drulhe, Monique Membrado, Jean Mantovani et Jean-François Barthe sont les fondateurs de cette école toulousaine qui fait actuellement autorité quant à la déprise. Sous leur plume, le concept est défini comme un «processus de réaménagement de la vie » (Clément et Mantovani, 1999) selon les modifications corporelles et relationnelles qui émergent au fil de l'âge. La prise en compte des capacités individuelles, des relations interpersonnelles, du parcours de vie antérieur et du contexte socioculturel permet au concept de déprise de saisir l'expérience individuelle du vieillissement, telle qu'elle se retranscrit dans les propos rapportés par les personnes vieillissantes elles-mêmes et / ou leurs proches, tout en considérant leurs activités (Drulhe et Pervanchon, 2004), leurs rapports au temps (Clément, 1994 ; 2000 ; Membrado et Salord, 2009), à l'espace, aux objets (Membrado, 1998 ; Membrado, Mantovani et Clément, 2008 ; Membrado et Rouyer, 2013), aux autres (Drulhe et al., 2007) et à soi (Clément et al., 1998 ; Mantovani et Membrado, 2000 ; Meidani, sous presse). Ces différents registres, conçus comme analyseurs de la déprise, proposent un ancrage pragmatique du concept et mettent en jeu les stratégies d'adaptation déployées par les personnes vieillissantes, susceptibles de préserver leur intégrité identitaire. De telles stratégies impliquent la sélection des activités, des objets, des lieux, des temps et des liens, et procèdent par suppression et / ou report sur d'autres registres équivalents : d'autres activités, objets, spatialités, temporalités ou réseaux relationnels.

La valeur heuristique de la déprise a déjà été testée à partir de nombreux programmes de recherche, nationaux et internationaux, portant sur la vieillesse et le vieillissement. Citons pour l'essentiel : les pratiques de la ville (Clément, Grand et Grand-Filaire, 1996), la secondarité (Membrado, 1998), l'habitat social (Clément, Mantovani et Membrado, 1998), les âges du chez-soi et l'habiter (Membrado, Mantovani et Clément, 2008 ; Membrado et Rouyer 2013), la conduite automobile (Drulhe et Pervanchon, 2004), les usages des objets techniques dans la sphère domestique (Clément et al., 1999), les temps du mourir (Clément, 1994 ; Clément, 2000 ; Meidani, sous presse), les transports urbains (Clément, 2006), l'expérience du voisinage (Drulhe et al., 2007), l'interface entre aide formelle et aide informelle (Clément et al., 1998 ; Clément et Lavoie, 2001 ; 2005), les expériences temporelles au grand âge (Membrado et Salord, 2009), les dispositifs de prise en charge en gériatrie (Clément, Mantovani et Membrado, 2004), le vieillissement des migrants (Rafaï et al., 2007), ou encore, les enjeux de santé et les formes de médicalisation en gérontologie avec une attention soutenue au corps vieillissant et à la maladie d'Alzheimer (Drulhe, 1993 ; Drulhe et Clément, 1998 ; Meidani et Membrado, 2011 ; 
Rolland-Dubreuil, 2003 ; Meidani, 2007 ; 2014 ; 2015 ; sous presse). Le concept, au cours de son élaboration et de son histoire, a été enrichi et mobilisé par l'apport de diverses disciplines et professions tels que les gériatres, les géographes ou les linguistes.

À partir de cette œuvre pionnière des auteurs de l'école toulousaine, d'autres chercheurs ont cherché à mobiliser le concept de déprise dans leurs travaux (Mallon, 2001 ; Caradec, 2001 ; 2004 ; Pennec, 2012 ; Meidani et Membrado, 2011). Leurs contributions ont permis à la fois de systématiser la réflexion autour de cet outil conceptuel, de le promouvoir, mais aussi d'en esquisser les limites.

\section{Les grands axes théoriques du concept de la déprise}

L'objectif de ce numéro thématique est de discuter la pertinence, l'évolution et l'ancrage empirique du concept de déprise mais aussi de soulever toutes ces questions que la déprise pose sans y répondre. Avant de présenter les textes qui le composent, quelques précisions théoriques semblent nécessaires, à commencer par le contexte et la genèse du concept. La déprise a été forgée en réponse à deux grandes théories en gérontologie sociale, celle de l'activité (Havighurst et Albrecht, 1953) et celle du désengagement (Cumming et Henry, 1961). Elle s'oppose surtout à la seconde et s'en différencie dans la mesure où elle n'envisage pas le processus de vieillissement comme une série de pertes, un déclin progressif conduisant au retrait social. Au contraire, le concept de déprise permet de travailler l'expérience individuelle du vieillissement à travers un double processus, de pertes et de gains. Ce point de départ incarne un des atouts majeurs du concept et permet de mieux comprendre l'attention accordée aux stratégies adaptatives. En ce sens, le concept invite à prendre de la distance par rapport à une représentation déficitaire de la vieillesse.

\section{Au cœur des expériences plurielles du vieillissement}

Conçu comme une construction sociale plurielle, le vieillir épouse les formes hétéroclites de la déprise (Mantovani et Membrado, 2000) allant des déprises plus ou moins «inquiètes » - là où le sentiment de l'existence rencontre le sentiment de finitude - aux déprises « ultimes » qui conduisent les aînés à renoncer à des activités jugées essentielles pour elles. Complétant cette nomenclature, d'autres idéaux-types ont été proposés à l'image de la "déprise stratégique ", qui désigne des situations dans lesquelles les personnes vieillissantes se situent dans une démarche d'anticipation des effets du vieillissement (Mallon, 2001), ce qui n'est pas sans rappeler la logique de préservation de soi mise en exergue par Jean Mantovani et Monique Membrado (2000).

Si le choix du cadre de référence propre à une certaine forme de déprise apparaît plus au moins stabilisé à un moment donné du parcours de vie, il n'est pas exempt de possibles mutations : l'expérience du vieillir se déploie dans un rapport flottant entre ces différents registres. De même, les diverses formes de la déprise se déclinent différemment selon des appartenances socioculturelles multiples (genre, génération, niveau d'instruction, conditions de vie, etc.), mettant à l'épreuve la figure homogénéisante des «personnes âgées » promue par les politiques publiques et axée sur les pertes. En ce sens, le concept de déprise permet d'avancer vers une 
politique sociale de la vieillesse affranchie des considérations catégorielles et des logiques clivantes : seniors, $3^{\mathrm{e}}$ et $4^{\mathrm{e}}$ âge, " dépendants », etc.

On pourrait soutenir que la déclinaison de la déprise selon les formes qui viennent d'être présentées interroge la possibilité d'une gradation du mécanisme observé, ponctué par une tendance à la baisse des activités et un rétrécissement des réseaux relationnels et des espaces au cours de l'avancée en âge. Mais la déprise n'a rien d'un processus mécanique irréversible. Elle ne peut pas être définie comme une succession d'étapes prédéterminées qui aboutirait fatalement au stade final : la " déprise ultime " située au palier de la mort. Ce qui revient à dire que l'avancée en âge peut activer des formes de déprise sans que cette dynamique soit universelle ou systématique (Drulhe et al., 2007).

\section{La dynamique de la reprise à l'œuvre dans la déprise}

La déprise peut déboucher sur des rebondissements remarquables. Lors de ces reprises, guidées par une dynamique d'autodétermination, l'individu se rattache à des activités, des espaces, des temporalités, des objets, des liens ou en explore de nouveaux, ce qui lui permet de préserver une certaine familiarité avec le monde (Caradec, 2007). Et pourtant l'interprétation de la déprise sous l'angle de la reprise n'a pas toujours été développée dans la littérature dédiée à ce concept. la dynamique de rebond a été amplement investie dans les travaux de ses fondateurs. De quoi s'agit-il au juste ? En avançant en âge, les individus tentent de faire face à l'emprise institutionnelle, aux normes et aux valeurs qui l'animent, à l'avènement de la maladie et / ou à la disqualification sociale qui leur est infligée, en développant de la résistance visant à sauvegarder leur sentiment d'identité et leur autonomie. En ce sens, la reprise devient le signe d'un certain contrôle que chacun exerce sur sa vie (Drulhe et al., 2007). Au sein de ce processus on enregistre des ruptures qui deviennent de plus en plus nettes (scissions affectives, pertes de liens, ruptures dans sa trajectoire de santé, etc.). Toutefois, la déprise procède par étapes ou si l'on préfère par paliers, ce qui signifie que la dynamique qu'elle engendre n'est intelligible qu'à l'aune de ces reprises, conçues ici comme des entreprises de résistance négociées, qui permettent à l'individu de faire face aux difficultés qu'il rencontre.

Si le renoncement existe, il convient de souligner que ce qui anime la dynamique de la déprise c'est plutôt une logique de remplacement que d'abandon (Clément et Mantovani, 1999). Les choix ainsi opérés sont guidés par des changements - d'ordre corporel, psychique, cognitif, biographique, social - et visent à pallier à la fatigue éprouvée et / ou à l'absence d'envie, auxquelles vient s'ajouter la volonté de se consacrer à «ce qui compte » et fait encore sens pour soi. En mettant en place des stratégies d'accommodation, les personnes procèdent par anticipation: elles économisent leurs forces, se ménagent, réduisent la prise de risques, évitent des activités jugées secondaires ou trop coûteuses en énergie. En ce sens, la reprise est un hymne à l'inventivité déployée " pour contourner ces obstacles et leur substituer de multiples ouvertures » (Drulhe et al., 2007, 336-337).

Soyons clairs, il ne s'agit pas ici de présenter la reprise comme une "réponse en miroir " à la déprise mais de rappeler que le concept témoigne de la possibilité positive qu'a un individu à s'adapter à l'expérience qu'il fait du vieillissement. 


\section{Les « critères » de sélection des activités au sein du mouvement de la déprise}

La déprise procède par sélection. Mais comment s'opèrent ces choix ? Pour établir ses choix l'individu a besoin de critères qui lui permettent de se déprendre de certains domaines de son existence pour mieux rester accroché, investi ou impliqué dans d'autres.

Le premier critère renvoie au capital corporel et à la trajectoire de santé, plus ou moins lisse ou semée d'entraves et d'embûches. Au sein de cette lecture capacitaire, pouvoir et vouloir se donnent la main. II s'agit là de s'attarder sur la question de l'envie et du désir qui, affranchie des contraintes d'autrefois, vise à assurer un certain goût à la vie, tout en prenant en compte cette « énergie vitale » qui s'amoindrit avec le temps (Barthe, Clément et Druhle, 1988) et qui trouve son expression d'un côté, dans la fatigue éprouvée par les plus âgés, de l'autre, dans la conscience de la proximité de la mort.

Le second critère implique la proximité relationnelle et géographique à laquelle vient se mêler le principe de réciprocité (Mantovani et Membrado, 2000). Mais la logique sélective et les aménagements qui en découlent trouvent aussi leur source dans le parcours de vie de la personne qui oriente la sélection des activités avec toutes les modifications des rôles et des statuts qu'annoncent les grands changements de la vie (Cavalli, 2012).

Enfin, un dernier critère renvoie au contexte socioculturel, appréhendé tant à un niveau macrosociologique, par les politiques publiques en matière de vieillesse et les modèles normatifs qui les animent, qu'à un niveau méso-sociologique visant à situer l'individu dans de multiples collectifs d'appartenances et l'ordre institutionnel.

\section{Rapport d'extériorité et marges d'actions}

Les critères énumérés ci-dessus montrent que les expériences du vieillir impliquent toujours un rapport d'extériorité. Celui-ci épouse parfois des contours radicaux: " nous ne serons jamais ces vieux / ces vieilles que nous croisons sur notre chemin ». Cette manière de tenir à distance le portrait du « vieillard » n'est pas sans impact sur la construction sociale de l'expérience du vieillir. Refusant de s'identifier à cette figure dégradante, les personnes vieillissantes mettent en place des stratégies de contournement (Mantovani et Membrado, 2000). Ces stratégies se disent dans le refus d'adopter certains rôles, de recourir à certains objets, de pratiquer certaines activités, de se plier à certains temps institutionnels, ou encore, d'expérimenter certains espaces publics - une autre manière de tenir à l'écart l'épreuve de disqualification qui accompagne le vieillir.

Toutefois, ce rapport d'extériorité ne saurait se réduire à la place qu'occupe l'autre (autrui significatif ou pas) dans l'expérience individuelle du grand âge. Cette extériorité se traduit aussi dans les modèles, les normes et les valeurs actuelles qui se concrétisent dans le nouveau leitmotiv de notre époque : le «bien vieillir ». Le rapport au vieillissement donne à voir la multitude des modes de négociation du vieillir et exprime des définitions de soi diverses. Si ce rapport à l'extériorité oriente les façons dont les personnes expérimentent leur avancée en âge, il n'est pas suffisant pour épuiser leurs expériences individuelles (Mantovani et Membrado, 
2000). Les expériences du vieillissement se construisent certes avec mais aussi malgré, à côté ou contre les politiques publiques, les modèles normatifs et les représentations qui les traversent.

\section{De la préservation de soi à l'ancrage relationnel du concept}

De toute évidence la dynamique de la déprise qui caractérise le processus individuel de vieillissement, ne peut pas se réduire à son versant pragmatique, matérialisé à travers un réajustement des activités, des objets, des temporalités et des espaces. Les stratégies adaptatives qui travaillent les pratiques individuelles doivent aussi se comprendre dans les relations à autrui, qui participent à l'expérience individuelle du vieillissement et orientent les avatars identitaires. Les négociations du vieillir s'accomplissent en lien avec les autres (proches familiaux, voisins, amis, professionnels, etc.), montrant bien que les choix des personnes vieillissantes quant aux formes que prendra leur propre vieillissement (Clément et al., 1998) se réalisent dans un vaste réseau d'interdépendances (Clément, 2006).

Dans cette perspective, la déprise constitue un excellent levier d'autonomie (Clément, 1996 ; Mantovani et Membrado, 2000), puisqu'elle permet d'orienter les transactions qui s'opèrent entre les personnes vieillissantes et leur entourage proche, mais aussi avec les professionnels amenés à intervenir dans leur vie. C'est dans ce sens que la déprise répond également et, surtout, à une logique de préservation de soi qui vise à conserver un sentiment de continuité identitaire, tout en permettant aux personnes vieillissantes de s'expérimenter, de se vivre, de se déployer, dans d'autres registres que ceux réservés à la catégorie de "vieillard ». En effet, c'est en étant autre chose que "vieilles » qu'elles parviennent à défendre leur rôle d'acteur dans les négociations qui forment leur expérience de l'avancée en âge. Le concept de déprise permet d'appréhender l'ancrage relationnel de ces négociations et, plus généralement, de toutes expériences de vieillissement.

\section{L’intérêt du concept pour les professionnels de la gérontologie}

Aussi étudié soit-il, le concept de déprise nécessite d'être réinterrogé à l'aune d'un regard disciplinaire croisé. C'est l'un des objectifs de ce numéro, qui entend aussi donner à voir l'intérêt du concept pour les professionnels de la gérontologie, notamment dans l'univers des soins. En effet, l'expérience du vieillir est traversée par des figures d'aînés qui questionnent le sens des pratiques de soins et contribuent en retour à la construction d'une culture professionnelle qui gagnerait à intégrer le concept de la déprise, entendue comme une entreprise plurielle négociée, un travail d'adaptation et de réaménagement de l'existence. En interrogeant la place sociale et l'environnement relationnel des aînés, la déprise s'attarde sur le pouvoir décisionnel des soignés, considéré non seulement du point de vue de la personne vieillissante mais aussi de son entourage. Ce faisant, elle positionne les questions éthiques au centre des enjeux du care et du cure. Le défi devient alors l'élaboration d'un ordre concerté entre soignés et soignants qui échappe à l'emprise médicale. Par ailleurs, la déprise restaure la plus-value de l'expérience singulière du vieillir, rappelant que l'âge chronologique et les pertes capacitaires ne sont pas les indicateurs du vieillissement les plus pertinents. En ce sens, le concept incarne un marqueur dynamique du vieillissement. II éclaire aussi une question existentielle de premier ordre pour les personnes vieillissantes: la qualité du reste à vivre, considérée du 
point de vue du sujet. Le concept de déprise, affranchi des malentendus qui le confondraient avec un lâcher-prise, passe alors de l'heuristique à l'opérationnel, conduisant à une prise en compte des expériences singulières des aînés, situées dans leurs parcours de vie.

\section{Introduction des différents articles du numéro}

Dans le premier libre propos, Serge Clément, Marcel Drulhe, Jean Mantovani et Monique Membrado reviennent sur l'histoire du concept de déprise trente ans après son invention, et mettent en exergue le jeu de rapprochements et de distanciations avec la théorie du désengagement forgée par Elaine Cumming et William Henry en 1961 aux États-Unis. Le fondement interdisciplinaire du concept se déploie à travers sa genèse. C'est en cherchant à répondre aux questions des épidémiologistes que l'équipe toulousaine rencontre la valeur prédictive des facteurs dits subjectifs de mortalité : l'absence de projet, le sentiment d'inutilité, une auto-évaluation négative de la santé. C'est aussi ce même ancrage interdisciplinaire, nous montrent les auteurs, qui explique la trajectoire conceptuelle d'une notion née à la fin des années 1980 et qui restera en retrait quelques années avant d'être investie à nouveaux frais en 1995, sous la plume de Serge Clément, Jean Mantovani et Monique Membrado. Présentée alors comme une stratégie, parmi d'autres, permettant d'organiser son mode de vie - «être âgé et vieux à la fois » - le concept s'étendra vite à l'ensemble du processus de vieillissement. Durant cette même période, les modes de sélection des espaces fréquentés, des activités et des relations sont placés au cœur de l'analyse donnant à voir cette tension inhérente au processus de la déprise : « se ménager et se risquer » (Clément, Mantovani et Membrado, 1996) qui met en jeu la conservation de son identité. Si les premiers travaux d'élaboration du concept insistent sur la capacité réactive des acteurs, montrant que l'abandon des certaines activités reconnues comme structurantes se réalise sur fond de continuité d'un mode de vie jusqu'alors adopté, les auteurs donnent à voir que la suite des recherches sur la déprise ne manquera pas de souligner le regard d'extériorité qui caractérise ce processus. S'attardant sur l'environnement social mais aussi sur les appartenances sociales multiples de la personne, la déprise se déploie dans sa pluralité. La typologie qui en résulte rejoint celle des formes du vieillir. C'est alors que la notion de la reprise prend place, tandis que l'apport des travaux interactionnistes fait valoir le vieillissement comme un processus négocié plutôt qu'un état imposé. Au début des années 2000, Vincent Caradec et Isabelle Mallon proposent la mise à l'épreuve du concept dans un mouvement d'extension dans le champ de la sociologie du vieillissement initié par son adoption par d'autres chercheurs. Si le fondement est sociologique, le retentissement dans les modèles analytiques de psychologues (Baltes et Baltes, 1990) apporte la preuve d'un paradigme discuté au sein de l'ensemble plus vaste des gérontologues, ce qui a permis d'affiner son intérêt et d'asseoir la nécessité de poursuivre sur cette voie originale.

L'article d'Éric Gagnon rend compte de la ligne de tension qui traverse le processus de déprise, déjà mise en évidence par ses fondateurs, et qui oscille inlassablement entre restriction des capacités individuelles et ressources disponibles insoupçonnées. L'amphibologie qui en résulte incite l'auteur à penser ensemble autonomie et dépendance, permanence de soi et mutation identitaire, humanité / socialité et finitude. Si l'analyse proposée souligne la place qu'occupent l'environnement de la personne vieillissante et les stratégies de reconversion dans le 
processus de déprise, elle fait aussi place à leur valeur symbolique. Gardant à distance une lecture fonctionnelle de la déprise, le texte met en évidence toutes ces dimensions du social auxquelles le concept se fait écho : positionnement dans la structure sociale, rapports sociaux, réseaux d'échanges. S'attardant dans un premier temps sur la notion de dépendance, l'auteur montre que c'est dans l'interdépendance que l'autonomie se déploie. Plus ou moins visibles, minimisées, stigmatisées ou valorisées, les formes de dépendance qui en découlent se déclinent différemment selon les services octroyés, les liens sur lesquels ils prennent appui et les activités rendues ainsi possibles. Glissant progressivement des questions d'autonomie vers celles d'identité, la réflexion proposée montre en quoi la déprise interroge la dynamique du changement identitaire. Dans un rapport circulaire entre maintien de soi et mutation, elle permet d'éclairer ces dimensions identitaires de soi que les personnes souhaitent préserver et ce qu'elles consentent à changer. Examiné à l'aune de la déprise, le vieillissement se révèle alors pluriel. Mais la déprise pose aussi la question du temps long et, par ricochet, ouvre sur d'autres dimensions: celles de la mort, de la socialité et de l'humanité. En avançant dans l'âge, les personnes s'éloignent progressivement du monde des vivants pour se rapprocher de celui de leurs ancêtres. La déprise incarne alors une forme de défense de soi qui prend congé des injonctions d'un monde dans lequel on ne se retrouve plus tout à fait. De la sorte, la déprise donne le ton de notre vision de la mort. En conclusion, Éric Gagnon montre comment les notions d'autonomie, d'identité mais aussi la mort, font retour sur le concept de la déprise favorisant ainsi une critique de ses limites et mettant en évidence toutes ces interrogations que la déprise laisse ouvertes.

C'est à la lumière philosophique que Pierre Ancet explore le rapport que la déprise entretient avec le récit de soi, conçu ici comme vecteur de continuité identitaire. L'auteur examine l'hypothèse selon laquelle le récit favoriserait le processus de déprise et rend compte des obstacles de cette entreprise. L'analyse part de la thèse de l'identité fictive de Paul Ricœur et avance l'idée que la fiction participe de la déprise, appréhendée comme mouvement incessant entre le renoncement, la résilience et l'adaptation. Lorsque la déprise est source de rebond, elle s'apparente à un renoncement réfléchi qui signe la reconnaissance de soi dans ce travail qui permet de se reprendre. Selon l'auteur, la déprise s'inscrit dans le récit lorsque la conscience d'un temps dorénavant compté se fait jour. En examinant les difficultés du récit de rendre compte de la déprise, Pierre Ancet illustre son propos en convoquant la sphère des soins. II examine tour à tour les contraintes organisationnelles de l'univers des soins, l'emprise de la douleur, la qualité de l'accompagnement mais aussi l'objectivation qui va de pair - obstacles redoutables à la déprise stratégique que le récit de vie peut envisager. Si le cumul d'obstacles met à l'épreuve le travail de production de soi dans le récit, le processus de déprise ne s'annule pas pour autant. Même les narrations les plus marginales où la falsification permet d'inventer une identité fictive suscitée alors sous l'emprise de la maladie démentielle, rendent compte d'une forme de déprise radicale et subie, et en cela elles doivent être considérées dans l'analyse du spectre d'intelligibilité que la déprise initie.

Si les travaux sociologiques disponibles sur la déprise permettent d'appréhender le processus à travers lequel les personnes avançant en âge négocient leur vieillissement, force est de constater que la littérature gérontologique portant sur ce concept est restée taciturne sur les effets de la déprise sur l'entourage, notamment 
professionnel, des aînés. Partant de ce constat, Véronique Feyfant se propose de considérer comment le processus de déprise retentit sur les pratiques professionnelles des infirmières libérales. C'est à partir d'une recherche ethnographique portant sur ce corps de métier et les interventions auprès de personnes qualifiées des dépendantes que l'auteure examine le rôle de la déprise dans l'élaboration de leurs pratiques professionnelles. Véronique Feyfant s'attarde sur le cas emblématique d'un de ses enquêtés, Antoine, qui suite à une hospitalisation est amené à apporter la preuve de sa capacité à rester seul chez-lui. La vision de l'autonomie exposée par Antoine froisse les infirmières et tient à distance les définitions des sociologues. En composant avec les contraintes imposées par les professionnelles, notamment en termes d'horaire ou de qualité des soins, Antoine s'adonne à un travail de recomposition des liens qui procède par tri relationnel et compose avec l'emprise institutionnelle à laquelle il est confronté. Dans un mouvement de co-construction qui se dit dans les stratégies de reconversion mises en place par réaction à des contraintes, Antoine fait preuve d'une réaction qui relève de la déprise dans ce que ce concept contient d'inventivité et de participation active, et avance dans un travail laborieux de reconstruction identitaire inscrite dans la relation aux autres. Ainsi l'auteure met en évidence les liens qui se tissent entre la déprise et les pratiques professionnelles de ces infirmières en montrant comment les soignés participent à la construction personnalisée du métier de ces soignantes dans un jeu interactionnel qui dénote des effets mutuels de la déprise tant sur la trajectoire individuelle des soignés que sur la trajectoire professionnelle des soignants.

C'est à partir d'une enquête par observation participante au sein d'une maisonnée, conduite sur trois années, que Françoise Le Borgne-Uguen et Simone Pennec rendent compte des supports sociaux qui soutiennent les possibilités de prises sur le monde. Examinant l'expérience de l'avance en âge d'un des membres de la maisonnée atteint d'une pathologie grave et chronique et des séquelles résiduelles induites par son état de santé, l'analyse proposée met en exergue un processus de déprise étayé par une entreprise concertée entre la personne malade, ses proches aidants et les professionnels. La polyvalence et la malléabilité du soutien pratique et symbolique apporté par le collectif de la maisonnée illustrent la dynamique de rebond et les stratégies déployées par les proches familiaux vis-à-vis des professionnels. Les auteures mettent en jeu l'économie domestique et morale de la maisonnée, alors que les positions statutaires des proches permettent de remanier les biens et les services apportés au malade. Après avoir présenté les formes de l'entraide opérantes au sein de la maisonnée et les tensions qui traversent cette entreprise de réaménagement, Françoise Le Borgne-Uguen et Simone Pennec s'attardent sur les pratiques quotidiennes des membres de la maisonnée. Le maintien d'un mode de vie ordinaire chez-soi est surtout rendu possible grâce à l'engagement des proches. Quant aux interventions des professionnels, elles permettent de remédier aux pertes fonctionnelles que le malade enregistre. L'enjeu de la déprise devient alors l'invention et la mise en place des nouveaux arts de faire, en continuité avec les valeurs d'avant la maladie. Au sein du couple, la conservation des normes de régulation advient comme un enjeu fort, un régulateur des formes de déprise. Oscillant entre les activités des soins de première recours et un travail de coordination de ces mêmes activités, l'engagement de l'épouse occupe ici une place centrale. Cette dernière se retranscrit aussi dans la préservation des sociabilités et les efforts de la conjointe d'épargner son époux de l'expérience du discrédit et de préserver la continuité identitaire, insistant sur ses habiletés maintenues. Alors même 
que la cohésion est mise en difficulté par les ressources limitées des ascendants, la dynamique ainsi dégagée donne à voir une forme de solidarité structurée par le parcours conjugal qui se déploie au sein d'un modèle de déprise communautaire et familialiste.

Laurent Nowik interroge les emménagements dans les habitats intermédiaires, complexes immobiliers dédiés aux personnes de plus de 60 ans, généralement associés à des services médico-sociaux accessibles. L'article prend appui sur une enquête par questionnaires adressés à plus d'un millier de personnes vivant en habitats intermédiaires. L'auteur aborde les raisons de ce choix résidentiel et les manières dont les personnes cherchent à s'approprier leur nouvel espace de vie et à préserver le sentiment du «chez-soi ». Mises en perspective à travers les ressources économiques et sociales dont disposent ces résidants, les raisons exposées semblent paramétrées par l'orientation de la sociabilité (plus ou moins chère à la sociabilité familiale) et par la quête des nouveaux appuis susceptibles de soutenir l'expérience du vieillir. L'analyse montre que les façons de vieillir en habitats intermédiaires constituent des illustrations du processus de déprise, dans tout ce que ce concept comporte de dynamique, allant des formes d'emprise jusqu'aux rebonds. Ainsi les personnes qui connaissent les problèmes de santé les plus graves semblent aussi sujettes à des attitudes de résignation et de repli, caractéristiques d'une déprise prononcée ou, pour ainsi dire, d'une emprise. Dans ces cas de figure où le déménagement s'organise souvent dans la précipitation, le choix résidentiel s'apparente plutôt à une rupture, une bifurcation plutôt imposée que choisie. En revanche, lorsque le choix résidentiel fait écho à une logique préventive qui procède par anticipation visant à retarder d'éventuelles pertes d'autonomie, les habitats intermédiaires représentent une prise sur le quotidien. Ici, la reprise est clairement identifiable, même si les soucis de santé peuvent venir contrarier cette perspective.

S'inscrivant dans une approche sociolinguistique, Alexandra Ortiz Caria met en lumière l'élaboration collective des interprétations situées de la déprise à partir d'une analyse conversationnelle, d'inspiration ethnométhodologique, des consultations filmées avec des personnes atteintes de troubles mnésiques ou d'une dégénérescence cognitive. La description de la déprise en termes capacitaires, identitaires ou d'autonomie est ici envisagée à travers l'interprétation que les soignants, formels et informels, réservent aux stratégies de reconversion mises en place par le patient dans un processus de déprise. Située au croisement de la lecture médicale et de l'expertise profane proposée par l'accompagnant (et à un moindre degré la personne malade), la déprise prend ici appui sur la nature des activités abandonnées et l'éventuel repli sur soi du patient. Plus ou moins socialement légitimes et justifiables, les déprises permettent alors, ou pas, de préserver l'identité de la personne malade et dénotent l'ancrage représentationnel de la démence. Quant à la lecture médicale de la déprise, négociée de façon située et traduite en signes cliniques, elle pose les indices de la normalité. Lorsque les patients arrivent à faire la démonstration d'une déprise qui évolue sur fond de continuité identitaire, les interactions des soins ouvrent sur une intelligibilité partagée qui repose pour l'essentiel sur des stratégies d'adaptation conçues comme des ressources. Au contraire, quand la dynamique de rebond et de préservation de soi fait défaut, l'interprétation médicale de la déprise fait la part belle aux pertes et au renoncement, et peut initier une procédure de réparation alors mise en place par le médecin. Quoi qu'il en soit, la déprise à toutes fins sociales, diagnostiques ou évaluatives, analysée à travers l'ordre interactionnel du soin permet de considérer la nature éminemment 
intersubjective de ces entreprises négociées. En guise de conclusion, Alexandra Ortiz Caria montre que «ce qui se joue en consultation n'est pas toujours d'ordre médical ", les élaborations endogènes de la déprise désignant des façons de tenir à distance des catégorisations exogènes proposées par l'approche biomédicale de la démence.

Explorant les configurations de l'aide informelle apportée aux aînés, Alain Grand montre dans quelle mesure et de quelle manière ce support conditionne l'expérience du vieillissement et joue un rôle déterminant sur le processus de déprise. Pour déployer son propos, l'auteur prend appui sur deux enquêtes réalisées dans le sudouest de la France auprès des personnes vieillissantes et des proches aidants. Le concept de déprise renoue ici avec les notions de négociation, de contrat tacite et d'autonomie relationnelle. La prise en compte de la dynamique d'aide observée considère tant les personnes vieillissantes elles-mêmes que le vécu des aidants, ce qui permet de dégager quatre figures idéal-typiques mettant en jeu le couple aidant / aidé et le processus de la déprise. L'article souligne alors que dans les relations d'aide informelle, la force du contrat tacite et les capacités de négociation qui animent le couple aidant / aidé, au même titre que l'autonomie relationnelle, avancent comme des conditions de réalisation des différentes formes de déprise. Appréciant l'ensemble de ces composantes, les personnes vieillissantes se trouvent plus ou moins perturbées ou soutenues dans la gestion de leur déprise. C'est notamment par l'intermédiaire des réaménagements relationnels induits par le soutien apporté à la vieille personne que cette dynamique de l'aide informelle peut freiner et / ou précipiter certaines modalités du processus de déprise. Ainsi l'auteur démontre que lorsque l'autonomie relationnelle de l'aidé prend appui sur l'aliénation de l'aidant, les effets produits sur la déprise peuvent être dévastateurs - l'épuisement de l'aidant étant susceptible de mettre en péril une déprise jusqu'alors bien négociée initiant des risques de rupture.

Le numéro thématique se clôture par un second libre propos qui vise à donner une autre dimension au concept de la déprise. Vincent Caradec y revisite sa trajectoire de recherche à l'aune du concept de déprise, rend compte de l'intérêt herméneutique de cet outil analytique et fait état de ses limites. L'auteur situe l'émergence du concept au tournant du XXle siècle alors même que la recherche sociologique délaisse progressivement l'étude de la vieillesse au profit de celle du vieillissement. Dès lors les recherches qui considèrent la déprise soulignent la nature processuelle du vieillir dans une approche dynamique mettant au cœur de l'analyse la notion d'identité et la catégorie d'acteur. Au creux de ce changement de paradigme et à partir de l'exploration des limites du concept des transitions biographiques conçues comme des étapes du vieillissement, l'auteur s'orientera vers le concept de la déprise cherchant à rendre visibles des mouvements constitutifs de cette expérience individuelle qui avancent masqués tels que le rapport au corps et le rapport au temps. II met en évidence les " déclencheurs » de ce processus (Caradec, 2007) systématisant de la sorte une réflexion dense, mais éparse, constitutive des travaux toulousains. Identifiant dans le préfixe privatif dé- une faiblesse qui alimenterait les malentendus, et soucieux de caractériser aussi finement que possible le vieillissement individuel, Vincent Caradec inspiré des travaux de Danilo Martuccelli (2006) se propose d'appréhender le vieillissement en termes d'épreuve. En englobant le concept de déprise, l'auteur cherche à mettre l'accent sur la dynamique de reconversion et le maintien de prises, et faire taire la dynamique du déclin. En 
parallèle l'analyse proposée fait place aux tensions identitaires, explore le maintien du pouvoir décisionnel et pose la question de l'autonomie. L'article conclut sur une autre stratégie analytique qui, à partir des catégorisations médicales ou politiques pourrait engager une approche intersectionnelle du vieillir éclairant de la sorte les effets produits par les rapports de domination, placés en retrait dans le paradigme de la déprise, tout en pointant les écueils d'une telle démarche.

\section{En conclusion}

Nous avons jugé judicieux d'engager une réflexion et de contribuer à une compréhension des débats actuels autour du concept de déprise, situés dans le champ de la vieillesse et du vieillissement. En accord avec cet objectif et en en faisant le point de départ de leur réflexion, les auteurs qui ont contribué à ce numéro thématique adoptent une posture originale et inédite et montrent en quoi le concept de déprise permet d'ouvrir le regard sur les expériences du vieillir. Portée par différentes disciplines, la lecture proposée donne alors à voir le caractère opérationnel du concept et invite à son usage, présentant l'intérêt et l'apport de l'analyse du processus individuel de vieillissement à travers la déprise. Au sein de cette réflexion, la plus-value de ce concept est considérée au même titre que ses limites, pour l'essentiel cristallisées autour de son préfixe privatif dé-. On pourrait entrevoir dans ce préfixe une faille, signe par excellence de l'asthénie conceptuelle de la déprise, comme on pourrait y identifier ce qui lui confère toute sa richesse. Quoi qu'il en soit, on ne pourra pas, au nom des ambitions analytiques aussi dignes soient-elles, priver le concept de son ancrage empirique, source d'inspiration et réalité observée par ses fondateurs. Tout comme on ne pourra pas nier que le potentiel analytique d'un concept n'est pensable qu'à l'aune de ce qui lui fait obstacle et qu'il cherche à dépasser. Les limites conceptuelles de la déprise ne se rendent visibles que dans les efforts fournis par les chercheurs qui la mettent à l'épreuve pour les surpasser, et toutes ces interrogations que la déprise soulève sans forcément y répondre en témoignent.

\section{Références}

Baltes, P. B. et Baltes, M. M. (1990). Psychological perspectives on successful aging: The model of selective optimization with compensation. Dans P. B. Baltes et M. M. Baltes (dir.), Successful aging: Perspectives from the behavioral sciences. (p.1-34). New York, NY: Cambridge University Press.

Barthe, J.-F., Clément, S. et Drulhe, M. (1988). Vieillesse ou vieillissement? Les processus d'organisation des modes de vie chez les personnes âgées. Les Cahiers de la Recherche sur le Travail Social, 15, 11-31.

Caradec, V. (2001). Sociologie de la vieillesse et du vieillissement. Paris, France: Nathan.

Caradec, V. (2004). Les 'supports' de l'individu vieillissant. Retour sur la notion de 'déprise'. Dans V. Caradec, et D. Martuccelli (dir.), Matériaux pour une sociologie 
de l'individu. Perspectives et débats. (p. 25-42). Villeneuve d'Ascq, France: Presses Universitaires du Septentrion.

Caradec, V. (2007). L'épreuve du grand âge. Retraite et société, 52, 11-37. Repéré à : http://www.cairn.info/revue-retraite-et-societe-2007-3-page-11.html

Cavalli, S. (2012). Trajectoires de vie dans la grande vieillesse : rester chez soi ou s'installer en institution ? Genève, Suisse : Université de Genève.

Clément, S. (1994). Les temps du mourir : changements et permanence. Cahiers Internationaux de Sociologie, XCVII, 355-371.

Clément, S. (1996). L'aide informelle visible et invisible. Dans S., Ayme et al., Handicap et vieillissement: politiques publiques et pratiques sociales (p.197204). Paris, France : INSERM.

Clément, S. (2000). Vieillir puis mourir. Prévenir, 38, 189-195.

Clément, S. (2006). Transports urbains et vieillissement. Informations sociales, 2(130), 72-79.

Clément, S., Drulhe, M., Dubreuil, C., Lalanne, M., Mantovani, J. et Andrieu, S. (1999). Les produits techniques dans les échanges entre les vieilles personnes, leur entourage et les services à domicile. Paris, France : Rapport MiRe-CNAV.

Clément S., Drulhe M., Durand D. et Membrado M. (dir.), (1998). Formes et sens du vieillir. Prévenir, 35, 5-8.

Clément, S., Grand, A. et Grand-Filaire, A. (1996). Aide aux personnes vieillissantes. Dans J-C. Henrard, S. Clément et F. Derriennic (dir.), Vieillissement, santé, société. (p. 163-189). Paris, France : Éd. INSERM.

Clément, S. et Lavoie, J.-P. (2001). L'interface aide formelle-aide informelle : au confluent de rationalités divergentes. Dans J.-C. Henrard, O. Firbank, S. Clément, M. Frossard, J.-P. Lavoie, A. Vezina, Personnes âgées dépendantes en France et au Québec. Qualités de vie, pratiques et politiques (p. 98-119). Paris, France : Éd. INSERM.

Clément, S. et Lavoie, J.-P. (dir.), (2005). Prendre soin d'un proche âgé. Les enseignements de la France et du Québec. Toulouse, France : Érès.

Clément, S. et Mantovani, J. (1999). Les déprises en fin de parcours de vie. Les toutes dernières années de la vie. Gérontologie et société, 22(90), 95-108.

Clément, S., Mantovani, J. et Membrado, M. (1995). Vieillissement et espaces urbains modes de spatialisation et formes de déprise. Recherche financée par le PIRVILLE-CNRS. Toulouse, France : C.I.E.U.

Clément, S., Mantovani, J. et Membrado, M. (1996). Vivre la ville à la vieillesse : se ménager et se risquer. Les Annales de la Recherche Urbaine, 73, 90-98. doi :10.3406/aru.1996.2010

Clément S., Mantovani J. et Membrado M. (1998). Expériences du vieillissement et formes urbaines. Dans N. Haumont (dir.), L'urbain dans tous ses états. Faire, vivre, dire la ville. (p. 231-242). Paris, France : L'Harmattan.

Clément, S., Mantovani, J., et Membrado, M. (2004). Du bon voisinage aux solidarités de proximité. Dans P. Pitaud (dir), Solitude et isolement des 
personnes âgées. L'environnement solidaire. (p. 105-138). Toulouse, France: Érès.

Cumming, E. et Henry, W. E. (1961). Growing old. The process of disengagement. New York, NY: Basic Books.

Drulhe, M. (1993). Effets de la culture sur les représentations et les activités des corps vieillissants. Dans C. Clanet, R. Fourasté et J.-L. Sudres (dir.), Corps, cultures, thérapies. (p. 265-284). Toulouse, France : Presses Universitaires du Midi.

Drulhe, M. et Clément, S. (1998). Enjeux et formes de la médicalisation : d'une approche globale au cas de la gérontologie. Dans $P$. Aïach et $D$. Delanoë (dir.), L'ère de la médicalisation. Ecce homo sanitas. (p. 69-96). Paris, France : Economica.

Drulhe, M., Clément S., Mantovani J. et Membrado M. (2007). L'expérience du voisinage : propriétés générales et spécificités au cours de la vieillesse, Cahiers internationaux de sociologie. 2(123), 325-339. doi : https://dx.doi.org/10.3917/cis.123.0325

Drulhe, M. et Pervanchon, M. (2004). Le vieillissement à l'épreuve de la conduite automobile : s'arrêter au nom de la santé ? Dans F.-X. Schweyer, S. Pennec, G. Cresson (dir.) Normes et valeurs dans le champ de la santé. (p. 91-103). Rennes, France : Éditions ENSP.

Havighurst, R. et Albrecht, R. (1953). Older People. New York, NY: Longmans \& Green.

Mallon, I. (2001). La recréation d'un chez soi par les personnes âgées en maison de retraite (Thèse de doctorat de sociologie). Université de Paris V-René Descartes, Paris, France. Repéré à : http://www.theses.fr/2001PA05H051

Mantovani, J. et Membrado, M. (2000). Expériences de la vieillesse et formes du vieillir. Dans Vieillir : I'avancée en âge. Informations Sociales, 88, 10-17.

Martuccelli, D. (2006). Forgé par l'épreuve. L'individu dans la France contemporaine. Paris, France : Armand Colin.

Meidani, A. (2007). Les fabriques du corps. Toulouse, France: Presses Universitaires du Midi.

Meidani, A. (2014). Alzheimer's Disease, Patients, and Informal Caregivers: Patterns of Care in France, Sweden, and Greece. Dans A.K. Leist, J. Kulmala et F. Nyqvist (éds.), Health and Cognition in Old Age: From Biomedical and Life Course Factors to Policy and Practice, International Perspectives on Aging. ( $\mathrm{p}$. 201-217). Cham, Suisse : Springer.

Meidani, A. (2015). À l'intersection du public et du privé : la médicalisation du grand âge à travers la prise en charge de la maladie d'Alzheimer en France et en Grèce. Dans A. Meidani, É. Legrand et B. Jacques. La santé : du public à l'intime (p. 37- 54). Paris, France : École des hautes études en santé publique.

Meidani, A. (sous presse). Perdre la tête, vieillir et mourir : l'expérience de la mort chez les patients atteints d'Alzheimer en France, en Grèce et en Suède. Dans A. Meidani et J.Y, Bousigue (dir.), Vivre la mort. Toulouse, France: Presses Universitaires du Midi. 
Meidani, A. et Membrado, M. (2011). Vieillesses et vieillissement : quels enjeux de santé ? Dans M. Drulhe, et F. Sicot (dir.), La santé à cœur ouvert. (p. 231-254). Toulouse, France : Presses de l'Université du Mirail

Membrado, M. (1998). Processus de vieillissement et "secondarité ». Dans J. Yerpez (dir.), La ville des vieux. (p. 95-106). Paris, France : Éditions de l'Aube.

Membrado M., Mantovani, J. et Clément, S. (2008). Habiter et formes du vieillir : du domicile à l'espace public. Dans S. Guérin (dir.), Habitat social et vieillissement : représentations, formes et liens. (p. 145-158). Paris, France : La Documentation française.

Membrado, M. et Rouyer, A. (dir.) (2013). Habiter et vieillir. Vers de nouvelles demeures. Toulouse, France : Erès.

Membrado, M. et Salord, T. (2009). Expériences temporelles au grand âge, Informations sociales. 3(153), 30-37. Repéré à : https://www.cairn.info/revueinformations-sociales-2009-3-page-30.htm

Pennec, S. (2012). Les solidarités pratiques du soin au grand âge : entre inégalités familiales et précariat professionnel. Dans F. Le Borgne-Uguen et M. Rebourg (dir.) L'entraide familiale : régulations juridiques et sociale. Rennes (p. 255-279). France : Presses universitaires de Rennes.

Rafaï, K., Mantovani, J., Duchier, J. et Gayral-Taminh, M. (2007). Le vieillissement des migrants, situation en Midi-Pyrénées. Rapport ORSMIP, INSERM U558. Repéré à : https://orsmip.org/le-vieillissement-des-migrants-situation-en-midipyrenees/

Rolland-Dubreuil, C. (2003). Vieillir en famille avec la maladie d'Alzheimer, Empan, 4(52), 107-115. doi : 10.3917/empa.052.0107

Emails auteurs : ameidani@univ-tlse2.fr ; stefano.cavalli@supsi.ch 